\title{
MEMS-Based Flow Controller for Flow Cytometry
}

\author{
Eugen Cabuz, Jay Schwichtenberg, Bob DeMers, Ernie Satren, Aravind Padmanabhan, \& Cleo Cabuz \\ Honeywell Intl. \\ 12001 State Highway 55, Plymouth, MN 55441, aravind.padmanabhan@honeywell.com
}

\begin{abstract}
The paper reports a highly miniaturized, low-power/low-cost micro flow controller capable of producing pressure-driven, pulsefree liquid flows in the $\mathrm{nL} / \mathrm{sec}$ to $\mathrm{mL} / \mathrm{sec}$ range. The system operates in closed-loop and relies on two high speed, low power microvalves and a newly developed, highly sensitive liquid flow sensor. A manually pressurized chamber is used as the pressure source. The system has been developed for providing the flows required in a portable flow cytometer [1] using hydrodynamic focusing but it can also be used as a general-purpose flow-driving module in a variety of microfluidic applications where small size, low-cost and low-power are essential. Point-of-care medical microinstruments, highly parallel processing in drug discovery applications, micro dosing system for drug delivery, on-line process monitoring in food/chemical industry are just some areas that would benefit from the described system. The flow controller is also ideally suited for operation in corrosive and biological fluids.
\end{abstract}

\section{INTRODUCTION}

Existing precision fluid delivery systems are bulky, expensive, and typically employ high-power syringe pumps (one order of magnitude larger power) [2]. Such systems are not suitable for use in low-cost portable instruments. The existing electroosmosis/electrophoresis-based pumping systems require high voltages, tend to be invasive and are typically useful only for very low flow rates. The peristaltic (thermal/electrostatic) pumps generate pulses and do not result in precise flow rate control. A recently published pressure-based mass flow controller [3] is about the same size (per channel) as our controller but the lowest flow it can control is two orders of magnitude higher and it consumes five times more power than our controller. There is therefore a need for a miniaturized, pulse-free, and low-power pumping system for controlling flow rates in the $\mu \mathrm{L} / \mathrm{s}$ and $\mathrm{nL} / \mathrm{s}$ regimes. The new flow controller that is reported in this paper addresses this need.

\section{DESCRIPTION}

The micro flow controller employs two key MEMS-based technologies that have been developed at Honeywell (Figure 1): electrostatically-actuated silicon microvalves [4], and glass-based thermal microbrick ${ }^{\mathrm{TM}}$ flow sensors [5].
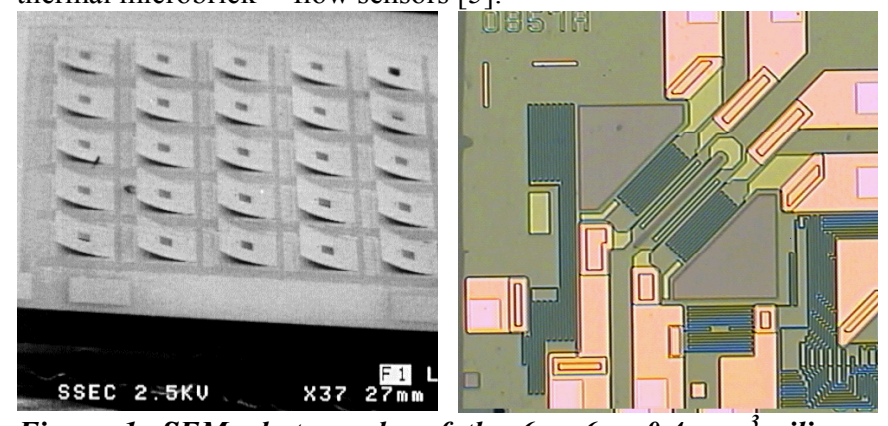

Figure 1. SEM photographs of the $6 \times 6 \times 0.4 \mathrm{~mm}^{3}$ silicon microvalve and $1.7 \times 1.7 \times 0.5 \mathrm{~mm}^{3}$ microbrick ${ }^{T M}$ liquid flow sensor chips.
Figure 2 shows a schematic structure of the proposed micro flow controller. The key elements/features of each channel of the controller are:

- $\quad$ an unregulated primary high pressure source $\left(\mathrm{P}_{\mathrm{d}}=0.2 \mathrm{psi}\right.$ gage; $P_{\max }=1.0$ psi gage; $\left.V_{\text {ol }} l_{\max }=2 \mathrm{~cm}^{3}=4 \times 6 \times 0.08 \mathrm{~cm}^{3}\right)$ which, for portable applications can be manually pressurized. A low power mesopump can be used to generate desired pressure in other cases [6];

- $\quad$ a regulated secondary pressure source (0.1-1 psi gage), with the pressure drawn from the primary pressure source and controlled through two low-power $(<1 \mathrm{~mW})$ \& fast $(\sim 1$ $\mathrm{ms}$ )/high impedance microvalves;

- a short response time ( $1 \mathrm{~ms})$ flow sensor capable of measuring liquid flows in the $\mathrm{nL} / \mathrm{sec}-\mathrm{mL} / \mathrm{sec}$ range;

- a closed loop circuit including the flow sensor and the valves that maintains a constant flow in each channel;

- a pneumatic-to-hydraulic interface realized with a porous plug;

- a very good seal between the flow controller and the fluidic channel;

- the overall response time for the flow controller is about 10 milliseconds.

\section{SIMULATION}

A SPICE system model has been developed to predict the operation of the flow controller and to study the effect of compliance in a miniaturized pneumatically driven pumping system. For the chosen microcytometer cartridge design, the model showed that the effect of vibrations on core formation and flow stability were either minimal or non-existent. This SPICE modeling result was confirmed by experimental work done with the flow controller. Detailed results of the simulation work will be reported at the conference.

\section{EXPERIMENTAL RESULTS}

The flow controller has been extensively characterized for different types of microvalves and different operation regimes. Flow rate pulsatility of less than 3\% (Figure 3) has been demonstrated for the microcytometer application.

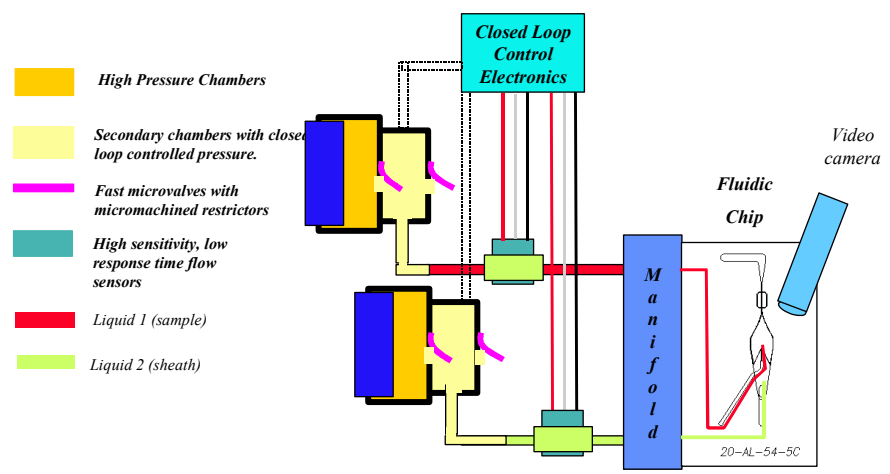

Figure 2. Schematic diagram of a 2-channel micro flow controller based on MEMS-based microvalves and flow sensors. 


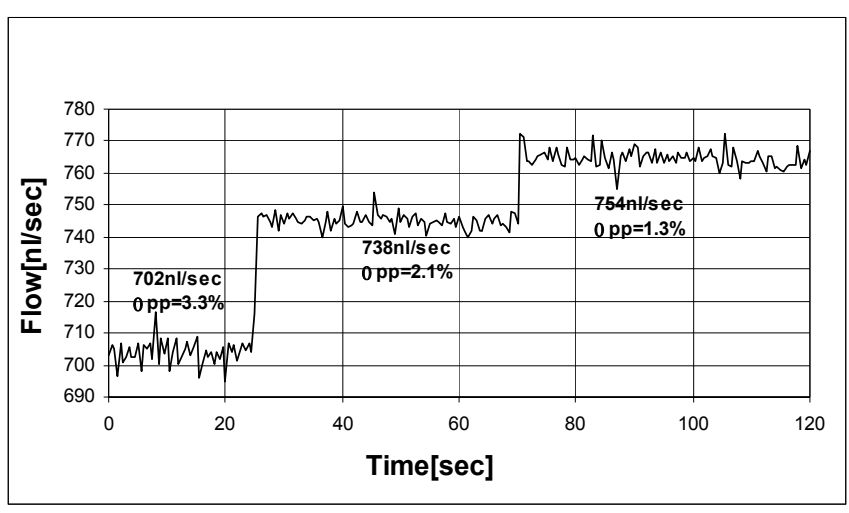

Figure 3. Experimental results showing excellent flow rate control (1.3 to $3.3 \%$ peak-to-peak rate variation) obtained by pneumatically driving the liquid from a manually pressurized source, through closed-loop control. Each closed-loop channel comprises of two silicon micromachined microvalves and a microbrick ${ }^{T M}$ liquid flow sensor.

This measured pulsatility is more than one order of magnitude better than what can be obtained using commercially available precision syringe pumps. A figure of merit defined as: size $\mathrm{x}$ cost $\mathrm{x}$ pulsatility for the proposed flow controller would be more than two orders of magnitude better than that for currently available, state-of-the-art open-loop fluid pumping systems.

Figure 4 shows a cross-sectional view through the five modules of the flow controller: pressure source module, microvalve module, reagent reservoir module, interface module, and flow sensor module.

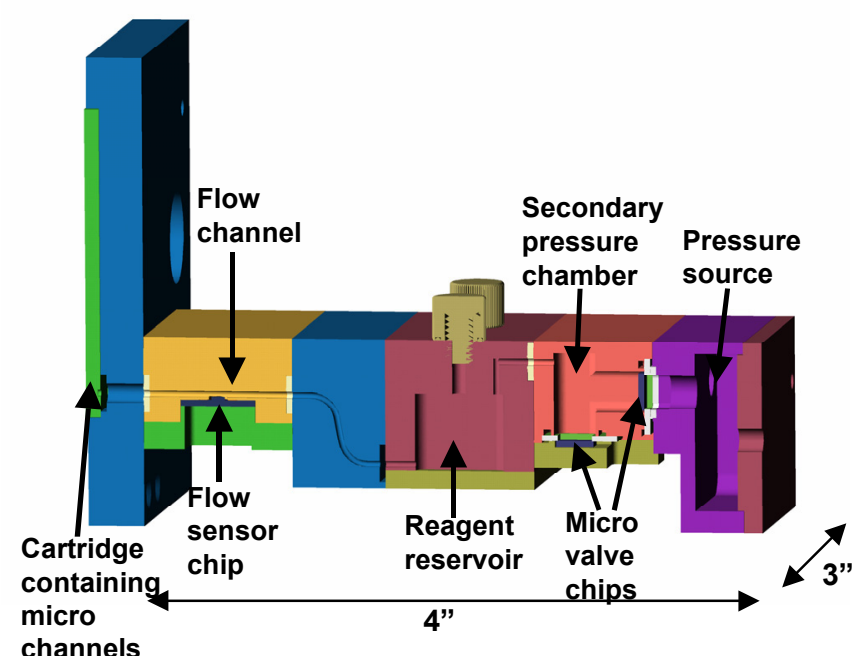

Figure 4. Cross-sectional view of the micro flow controller showing the various modules. The modules interface with each via custom-built polymer seals.

Figure 5 shows a photograph of the flow controller. This system was used to generate a $15 \mu \mathrm{m}$ wide core flow in the flow channel of the Honeywell microcytometer. Figure 6 shows the core widths that were generated for various sheath-to-core flow rate ratios. Stable, and repeatable core formation was demonstrated with the flow controller.

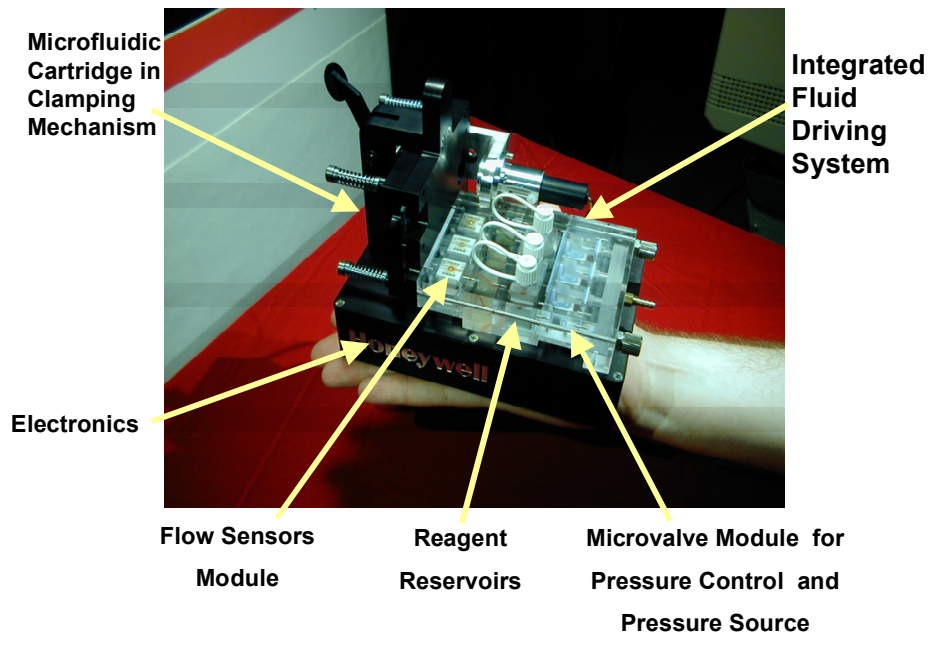

Figure 5. Photograph of the integrated version of the 3-channel micro flow controller showing its various components. The integrated flow controller is 4 " $x 3$ " $x 1$ ” in size.

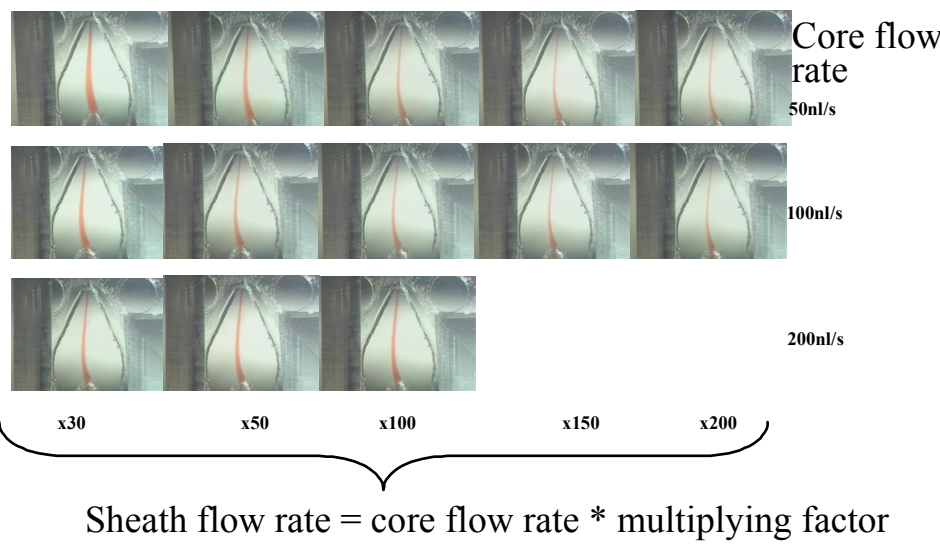

Figure 6. Optical images taken in the hydrodynamic focusing chamber of the flow cytometer showing the core flow (dark color) within the external sheath flow (colorless) for various sheath to core flow rate ratios. The flow controller produces a stable, pulse-free core flow for a range of sheath to core flow ratios.

\section{ACKNOWLEDGMENTS}

This work, done in collaboration with Micronics Inc., was supported by DARPA Contract MDA972-00-C-0029. Micronics designed/fabricated the microfluidic cartridges. We are grateful for the assistance of Curtiss Hella and the entire Micronics team.

\section{REFERENCES}

[1].[http://www.darpa.mil/MTO/bioflips/presentations/2001-1/index.html [DARPA BioFlip Program]

[2]. http://www.instechlabs.com/syringe.html [Harvard Apparatus Syringe Pump]

[3] Fitch, J., et al., "Pressure-based mass flow control using thermopneumatically actuated microvalves", Proceedings Sensors \& Actuators Workshop, Transducer Research Foundation, OH, pp. 162-165, 1998.

[4]. Ohnstein, T, et al., "Micromachined Silicon Microvalve", Proceedings of MEMS 1990, Napa Valley, CA, pp. 95-98, 1990.

[5]. Bonne, U., et al., "Microsensor Housing", US Patent 6,322,24, Issued November 27, 2001.

[6]. Cabuz et al, "Mesoscopic Sampler", Transducers'99, June 7-12, 1999, Sendai Japan 